\title{
Gray and white matter abnormalities in primary trigeminal neuralgia with and without neurovascular compression
}

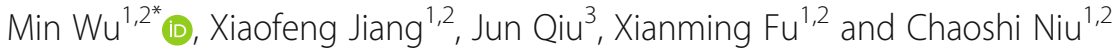

\begin{abstract}
Purpose: Previous researches have reported gray and white matter microalterations in primary trigeminal neuralgia (TN) with neurovascular compression (NVC). The central mechanism underlying TN without NVC are unknown but may include changes in specific brain regions or circuitries. This study aimed to investigate abnormalities in the gray matter (GM) and white matter (WM) of the whole brain and the possible pathogenetic mechanism underlying this disease.

Methods: We analyzed brain morphologic images of TN patients, 23 with NVC (TN wNVC) and 22 without NVC (TN wNVC) compared with 45 healthy controls (HC). All subjects underwent 3T-magnetic resonance imaging and pain scale evaluation. Voxel-based morphometry (VBM) and surface-based morphometry (SBM) were used to investigate whole brain grey matter quantitatively; graph theory was applied to obtain network measures based on extracted DTI data based on DTI data of the whole brains. Sensory and affective pain rating indices were assessed using the visual analog scale (VAS) and short-form McGill Pain Questionnaire (SF-MPQ).
\end{abstract}

Results: The VBM and SBM analyses revealed widespread decreases in GM volume and cortical thickness in TN wNVC compared to TN wONVC, and diffusion metrics measures and topology organization changes revealed DTI showed extensive WM integrity alterations. However, above structural changes differed between TN WNVC and TN woNVC, and were related to specific chronic pain modulation mechanism.

Conclusion: Abnormalities in characteristic regions of GM and WM structural network were found in TN woNVC compared with TN wNVC group, suggesting differences in pathophysiology of two types of TN.

Keywords: Trigeminal neuralgia, Voxel-based morphometry, Surface-based morphometry, Diffusion tensor imaging, Internal neurolysis, Neurovascular compression, Conditioned pain modulation

\section{Introduction}

Trigeminal neuralgia (TN), one of the most common pain conditions affect the craniofacial region, involves abnormalities in the peripheral and central trigeminal sensory pathways. The essential role of neurovascular

\footnotetext{
*Correspondence: wumin_ah@163.com

${ }^{1}$ Department of Neurosurgery, The First Affiliated Hospital of USTC (Anhui Provincial Hospital), Division of Life Sciences and Medicine, University of Science and Technology of China, Hefei, Anhui 230001, P.R. China

${ }^{2}$ Anhui Provincial Stereotactic Neurosurgical Institute, Hefei, Anhui 230001, P.R. China

Full list of author information is available at the end of the article
}

compression (NVC) in classical TN pathophysiology has been supported by the efficiency of microvascular decompression (MVD) in treating classical TN since 1960s [1], and the NVC has been considered as "target" for the treatment of TN with NVC (TN wNVC) [2]. Accumulating evidence suggests that $\mathrm{TN}$ wNVC and $\mathrm{TN}$ without NVC (TN woNVC), classically similar forms of pain manifestations, may nevertheless differ in pathogenic mechanisms. Several studies have consistently reported that TN occurs and recurs in the absence of NVC [3-5], while NVC of the trigeminal nerve (TGN) exists in a

C C The Author(s). 2020 Open Access This article is licensed under a Creative Commons Attribution 4.0 International License, which permits use, sharing, adaptation, distribution and reproduction in any medium or format, as long as you give appropriate credit to the original author(s) and the source, provide a link to the Creative Commons licence, and indicate if changes were made. The images or other third party material in this article are included in the article's Creative Commons licence, unless indicated otherwise in a credit line to the material. If material is not included in the article's Creative Commons licence and your intended use is not permitted by statutory regulation or exceeds the permitted use, you will need to obtain permission directly from the copyright holder. To view a copy of this licence, visit http://creativecommons.org/licenses/by/4.0/. The Creative Commons Public Domain Dedication waiver (http://creativecommons.org/publicdomain/zero/1.0/) applies to the data made available in this article, unless otherwise stated in a credit line to the data. 
sizeable population without TN [6]. There has been no recognized target for the treatment of $\mathrm{TN} w \mathrm{wVC}$, and patients with this type of TN have to underwent lesioning procedures, such as percutaneous balloon compression (PBC), percutaneous radiofrequency rhizotomy (PRR), or internal neurolysis (IN) [4, 7].

For some time, the abnormal neurovascular relationship in peripheral nerve system that trigger ascending changes in brain activity have been considered the foundation of TN wNVC pathophysiology and critical for the initiation and development of the pain attack events [8]. Studies have provided evidence that gray matter (GM) volume deficits and disruptions in the integrity of white matter (WM) have been observed in patients with CTN using structural and diffusion magnetic resonance imaging (MRI). According to a recent voxel-based morphometry (VBM) studies [9], the TN group showed significantly decreased gray matter volume in the bilateral superior/ middle temporal gyrus (STG/MTG), bilateral parahippocampus, left anterior cingulate cortex (ACC), caudate nucleus, right fusiform gyrus, and right cerebellum compared with the HC. In addition to GM volume deficits, alterations of WM integrity in the thalamicsomatosensory tract ipsilateral to the site of NVC was reduced in CTN patients via topographical analysis [10].

The previous research of our team has revealed microstructural alteration of the trigeminal nerve root in TN woNVC via DTI [11], and we planned to further our understanding of the central mechanisms of TN woNVC. This study thus aimed to concurrently investigate GM and WM abnormalities in TN wNVC and TN woNVC, and to explore microstructual similarities and differences between the two subtypes, even to deduce the causality between trigeminal sensory abnormalities and structural brain changes, and identify promising novel targets for TN woNVC treatment. We performed voxel-based morphometry (VBM) and surface-based morphometry (SBM) of structural MRI data and applied graph theoretical analysis and group connectometry analysis based on DTI data retrieved from a consecutive cohort of patients with TN wNVC or TN woNVC and healthy controls $(\mathrm{HC})$ in a single center database. To our knowledge, no previous study reports both alterations of GM volume and WM integrity in TN wNVC and TN woNVC patients, via VBM and SBM followed by diffusivities measures and graph theoretical analysis correlated to clinical covariates.

\section{Materials and methods Subjects \\ Inclusion and exclusion}

Study participants and data-acquisition This was a single-centre, retrospective study of patients treated surgically for CTN at Anhui Provincial Hospital between
2018 and 2020, approved by the Research Ethics Board of the Anhui Provincial Hospital. Diagnosis of CTN, defined as idiopathic, episodic, lancinating pain that lasted seconds, with pain-free episodes between attacks, by International Classification of Headache Disorders 3rd edition (ICHD-3) criteria, was the most important inclusion criteria. Potential study participants were identified from an operative database, and were included if they had undergone MVD or IN surgery performed by any one of two experienced neurosurgeons (Drs. X.-F.J. \& M.W.), had proof of successful technical completion of surgery according to operative notes. Patients with secondary factors may resulting in $\mathrm{TN}$, such as cranial tumors, multiple sclerosis (MS), or vertebrobasilar dolichoectasia resulting in brainstem compression, were excluded from this study [12]. Patients with a history of any previous non-TN neurosurgical procedures were excluded. Demographic and clinical data were obtained from physical and electronic patient charts. Patients were only included in the study once, even if they had undergone multiple $\mathrm{TN}$ procedures. In all cases, MRI images were collected within 1 week prior to the surgical procedure. Finally, a total of 23 patients with TN wNVC, 22 patients with TN wNVC and $45 \mathrm{HCs}$ were prospectively included in our study.

\section{Clinical characteristics and outcome assessment}

The following demographic and clinical data were collected: sex, age at preoperative MRI, duration of $\mathrm{TN}$, affected side, medications at time of preoperative MRI (carbamazepine or oxcarbazepine), and previous TN procedures. Duration of TN was defined as the amount of time between the dates of initial TN diagnosis to the date of preoperative brain MRI acquisition. Both the visual analog scale (VAS) and the short form of the McGill Pain Questionnaire (SF-MPQ) were applied for quantitative assessment of orofacial pain pre- and post-operatively, which represent the "sensory" and "affective" dimensions of pain severity respectively. Outcome were assessed from all patients in person at discharge (generally on postoperative day 7) and at the first outpatient visit (usually around postoperative day 50), which was used to calculate the average pain rating and obtain sensory, affective, and total pain indices.

\section{MRI data acquisition}

All subjects underwent MRI using the following imaging protocol: sagittal-oriented 3D T2-weighted images (TR/ $\mathrm{TE}=2500.0 / 244.6 \mathrm{~ms}$, field of view $(\mathrm{FOV})=256 \times 256$ $\mathrm{mm}^{2}$, with a $1 \mathrm{~mm}^{3}$ isotropic voxel size), sagittal-oriented 3D T1-weighted images $(\mathrm{TR} / \mathrm{TE}=8.6 / 4.0 \mathrm{~ms}, \mathrm{FOV}=256 \times$ $256 \mathrm{~mm}^{2}, 1 \mathrm{~mm}^{3}$ isotropic voxel size), and coronal-oriented $3 \mathrm{D}$ fluid-attenuated inversion recovery images $(\mathrm{TR} / \mathrm{TE}=$ $4800.0 / 266.8 \mathrm{~ms}$, FOV $=240 \times 240 \mathrm{~mm}^{2}$, a $1 \mathrm{~mm}^{3}$ isotropic voxel size). In addition, DTI scans were obtained for all 
subjects. DTI was performed using spin-echo single shot echo-planar pulse sequences with a total of 32 different diffusion directions (TR/ TE $=8620 / 85 \mathrm{~ms}, \mathrm{FA}=90^{\circ}$, slice thickness $=2.25 \mathrm{~mm}$, acquisition matrix $=120 \times 120$, FOV $=$ $240 \times 240 \mathrm{~mm}^{2}$, and b-value $\left.=1000 \mathrm{~s} / \mathrm{mm}^{2}\right)$. All of the scans were obtained using a 3T MRI scanner (Signa HDx; GE Healthcare; USA).

\section{T1 data processing}

VBM was performed with CAT12, an SPM12 extension with the default pipeline (http://dbm.neuro.uni-jena.de/ cat). This technique enables voxel-wise statistical analysis using the whole of automatically segmented gray and white matter. A unified segmentation approach was used. Subsequently, we used the affine registration algorithm to record all the native-space tissue segments to the standard Montreal Neurological Institute template (included in SPM12). The use of the exponentiated lie algebra toolbox (DARTEL) to all participants' GM and WM was necessary to refine the inter-subject registration via the application of the diffeomorphic anatomical registration. Images were also modulated with the objectives of preserving gray matter data and minimizing distortion of normalization. Finally, an 8-mm full width at half maximum Gaussian filter was applied to allow statistical analysis.

Surface-based morphometry (SBM) were also performed with CAT12. This type of SBM analysis employs an algorithm for automatic cortical thickness and central surface estimation. Topological correction and spherical mapping for inter-subject alignment and spherical registration were performed. Cortical thickness was estimated using a projection-based methodology by calculating the distance between the inner (boundary between white and gray matter) and outer (boundary between gray matter and cerebrospinal fluid) cortical surfaces. Finally, thickness meshes were smoothed with a $15-\mathrm{mm}$ Gaussian kernel, and a 20-mm kernel was used for the other parameters. The level of significance selected was uncorrected $p<0.001$, with no cluster-forming height threshold.

\section{DTI data processing}

Diffusivities measures were performed using DSI Studio (http://dsi-studio.labsolver.org). Fractional anisotropy (FA) measures directionality of water movement in brain tissues. The FA value was calculated using three eigenvalues of the diffusion tensor at each voxel. After creation of FA maps, the maps were smoothed (full-width half maximum $=8 \mathrm{~mm}$ ). Then, we segmented $\mathrm{GM}$ and WM from standard space. In addition, to minimize registration errors, we applied an additional FA threshold to WM with FA values lower than 0.2. Finally, we compared FA and radial diffusivity (RD) values at each voxel in WM. FA is highly sensitive for detecting microstructural changes in the WM; RD is a putative marker of myelination, whereas an increased RD is suggestive of de- or dys- myelination [13].

Graph theoretical analysis was also performed using DSI Studio. Nodes were defined as anatomical regions, and edges were defined by fiber density. Graph theoretical analysis was performed as follows. First, a tractography was generated from the DTI data, which entails reading and parsing DICOM files, image reconstruction to characterize the major diffusion direction of the fiber, and fiber tracking. Afterwards, the connectivity matrix was generated, which was calculated from the count of the connecting tracts. The Automated Anatomical Labeling (AAL) template was used as the brain parcellation, and every white matter fiber was evaluated to determine its extreme points. This step included acquiring a whole brain fiber track, which placed the seeding at the whole brain level, spatial normalization, and definition of the regions of interest, and building the connectivity matrix. At last, we calculated the global graph theoretical network measures from the connectivity matrix, including the mean clustering coefficient, characteristic path length, small-worldness, global efficiency, and local efficiency, to obtain quantitative information regarding the global network properties. In addition, we also obtained measures of strength, betweenness centrality, and eigenvector centrality to investigate changes in hub organization.

\section{MVD and IN}

Suboccipital retrosigmoid craniotomy and surgical corridor selection were performed in a manner previously described [14]. After complete exploration of the trigeminal root from the brainstem to the porus trigeminus of Meckel's cave, and verifying the presence or absence of NVC of whole segment of TR in posterior fossa. The manipulation of MVD was performed in the situation of NVC [14], while internal neurolysis was performed in the situation of absence of NVC, by Drs. X.F.J. and M.W. together, which had been published previously in detail by our team [15]. Both intraoperative findings and postoperative review of operative video were used to confirm the diagnosis of $\mathrm{wNVC} \mathrm{TN}$ or woNVC TN prospectively.

\section{Statistical analyses}

We employed two-sample $t$-tests (for age, sex, VAS, SFMPQ) and chi-square tests (for gender, affected side, antiepileptic drugs) to examine individual differences. To determine statistical significance, we used analysis of covariance controlling for age, gender, education, drink, smoke, hypertension, and diabetes mellitus. For VBM and SBM studies, group comparisons between patients 
Table 1 Demographic and clinical characteristics

\begin{tabular}{lllll}
\hline Variables $^{\mathbf{a}}$ & Healthy control $(\mathbf{N}=\mathbf{4 5})$ & TN wNVC $(\boldsymbol{N}=\mathbf{2 3})$ & TN woNVC $(\boldsymbol{N}=\mathbf{2 2})$ & Statistics $^{\mathbf{b}}$ \\
\hline Age, $\mathrm{y}$ & $49.36 \pm 11.58$ & $53.30 \pm 8.66$ & $47.77 \pm 9.24$ & $\mathrm{~F}=1.74, P=0.182$ \\
Sex (male / female), $\mathrm{n}$ & $22 / 23$ & $12 / 11$ & $8 / 14$ & $\mathrm{X}^{2}=1.31, P=0.520$ \\
Duration of illness, $\mathrm{y}$ & - & $5.74 \pm 3.35$ & $4.97 \pm 2.09$ & $\mathrm{t}=0.92, P=0.365$ \\
Side affected (L/R), $\mathrm{n}$ & - & $9 / 14$ & $10 / 12$ & $\mathrm{X}^{2}=0.18, P=0.668$ \\
VAS pain rating & - & $5.41 \pm 2.06$ & $4.48 \pm 1.54$ & $\mathrm{t}=1.65, P=0.106$ \\
SF-MPQ-Total & - & $13.13 \pm 4.99$ & $12.50 \pm 4.19$ & $\mathrm{t}=0.46, P=0.650$ \\
$\quad$ SF-MPQ-Sensory & - & $9.87 \pm 4.45$ & $6.77 \pm 3.87$ & $\mathrm{t}=2.48, P=0.017$ \\
$\quad$ SF-MPQ-Affective & - & $3.26 \pm 2.36$ & $5.72 \pm 1.75$ & $\mathrm{t}=3.97, P<0.001$ \\
Antiepileptic medication & - & & & $\mathrm{t}$ \\
$\quad$ Carbamazepine dose, mg/d & - & $9.13 \pm 5.24$ & $6.13 \pm 3.26$ &
\end{tabular}

${ }^{\mathrm{a}}$ Data are shown as mean $\pm \mathrm{SD}$ or $\mathrm{N}$

${ }^{\mathrm{b}}$ ANOVA, chi-square test, or independent t test

(all patients and those with and without NVC) and controls were performed using $\mathrm{T}$ contrasts to identify areas of increased and decreased abnormality, a $p$-value $<0.05$ was considered significant for all calculations. In the FA and $\mathrm{RD}$ value comparison, the statistical threshold for significance was $p$-value $<0.005$, uncorrected, and the minimum cluster size was set at 30 contiguous voxels. We investigated differences in the graph theoretical network measures between patients with $\mathrm{TN}$ and the $\mathrm{HCs}$, comparisons were analyzed with a Student's $t$-test; the
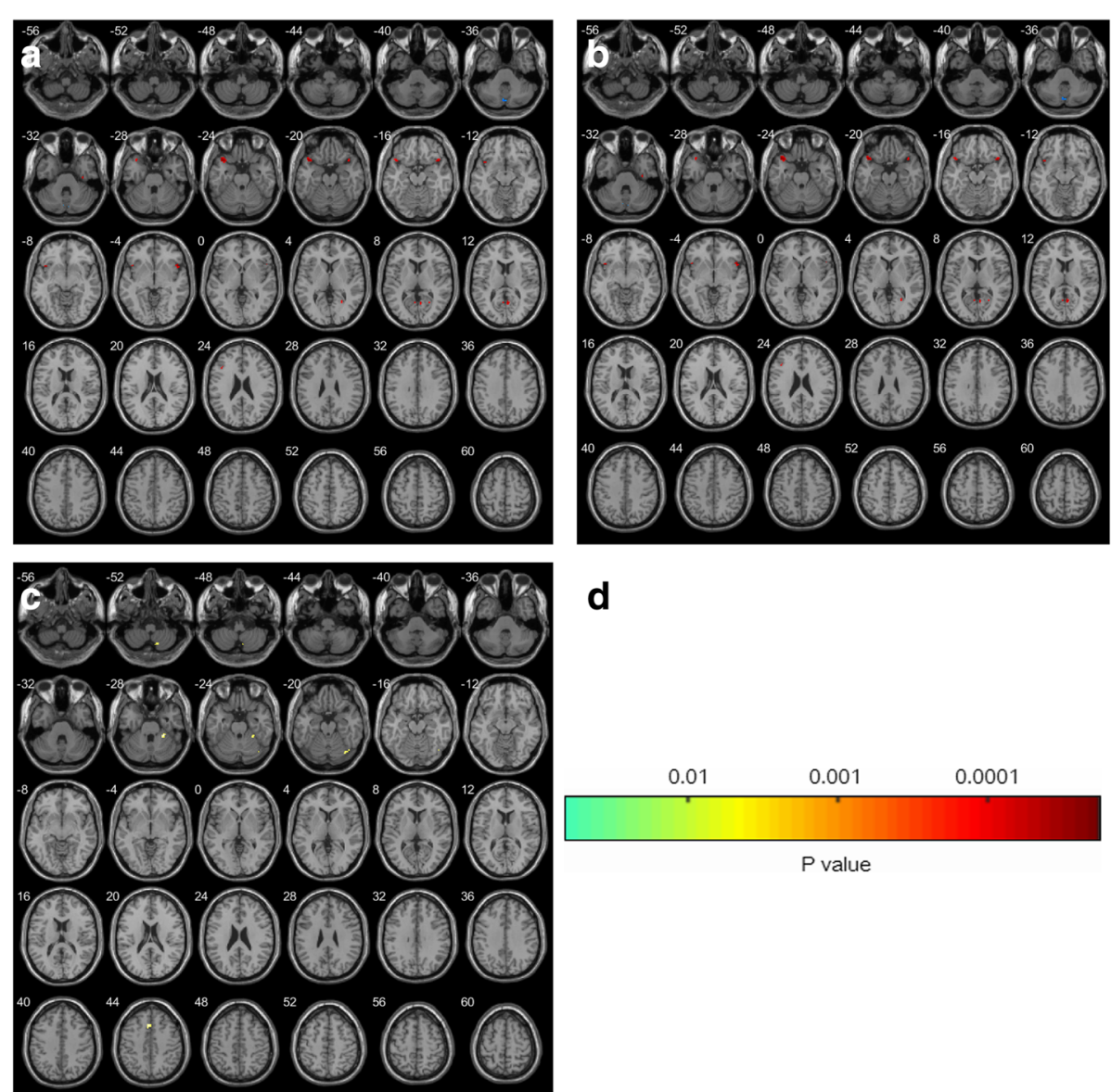

d

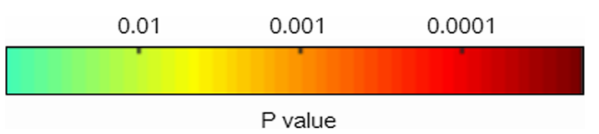

Fig. 1 Results of the voxel-based morphometry analysis comparing 23 patients with TN wNVC and 22 patients with TN wNVC and 45 healthy controls. Results of the statistical analysis are overlaid on an axial T1 anatomical magnetic resonance imaging template 
statistical threshold for significance was $p$-value $<0.001$. Statistical analysis was performed using the IBM SPSS Statistics ${ }^{\bullet}$ software (version 19.0).

\section{Results}

\section{Demographic and clinical characteristics of subjects}

Table 1 summarizes the demographic and clinical characteristics of the TN wNVC, TN woNVC and $\mathrm{HC}$ groups. There were no significant differences in age or sex among the 3 groups. In contrast to HCs, primary TN patients, displayed significantly higher VAS and SFMPQ index scores; however, no significant differences in pain rating indices between TN wNVC and TN woNVC. Treatment dose of antiepileptic drugs was also not significantly difference between TN wNVC and TN woNVC groups.

\section{Neuroimaging findings}

\section{Grey matter volume changes (VBM)}

Comparison of grey matter volumes in $\mathrm{HC}$ and CTN revealed significant regional grey matter volume changes in several regions (Fig. 1, Table 2). GM volume deficits were explored between the TN wNVC and TN woNVC groups. Compared to HC, TN wNVC showed GM volume reductions in multiple cortical and subcortical areas, including the frontal (right inferior frontal gyrus and left superior frontal gyrus), temporal (bilateral middle temporal gyrus), and parietal (bilateral posterior parietal cortex, left angular gyrus) areas, as well as in the left thalamus and right cerebellum. Peak voxel was observed in the left thalamus (Fig. 1a). However, TN woNVC showed GM volume deficits in bilateral inferior temporal gyrus, bilateral posterior cingulate cortex (PCC), and moreover, their cluster sizes were relatively small. Taken together, TN wNVC groups showed extensively asymmetry GM volume deficits, while TN woNVC groups showed bilaterally symmetric GM volume deficits (Fig. 1b). In addition, a direct comparison between TN wNVC and TN woNVC revealed significant difference in right medial prefrontal cortex (mPFC) and left cerebellum (Fig. 1c).

\section{Cortical thickness changes (SBM)}

In the comparison between TN wNVC and $\mathrm{HC}$, three clusters of decreased cortical thickness were detected by SBM; localized to the left inferior frontal gyrus, right medial frontal gyrus, bilateral superior occipital gyrus and periaqueductal gray (PAG) matter (Fig. 2a). In the comparison between TN woNVC and $\mathrm{HC}$, three cluster of decreased cortical thickness were detected, localized to the left angular gyrus, left medial frontal gyrus, left inferior temporal gyrus (Fig. 2b). However, in the direct comparison between TN wNVC and TN woNVC, two clusters of decreased cortical thickness were detected in TN woNVC, localized to bilateral mPFC (Fig. 2c) (Table 3).

\section{Diffusion value changes (DTI)}

Disruptions of WM integrity were explored in the TN wNVC and TN woNVC groups. Overall, wide-spread

Table 2 Pairwise comparisons of gray matter volume between TN WNVC, TN woNVC, and HC groups ${ }^{\mathrm{a}}$

\begin{tabular}{|c|c|c|c|c|c|c|c|}
\hline \multirow[t]{2}{*}{ Anatomical region } & \multirow[t]{2}{*}{ Side } & \multirow[t]{2}{*}{$\mathbf{T}$} & \multirow[t]{2}{*}{$P$} & \multicolumn{3}{|c|}{ Peak coordinates (MNI) } & \multirow{2}{*}{$\begin{array}{l}\text { Cluster } \\
\text { size } \\
\left(\mathrm{mm}^{3}\right)\end{array}$} \\
\hline & & & & $x$ & $y$ & $z$ & \\
\hline \multicolumn{8}{|l|}{ TN wNVC vs. HC } \\
\hline Thalamus & $\mathrm{L}$ & 5.45 & 0.017 & -17 & -21 & -6 & 4430 \\
\hline Superior frontal gyrus & L & 6.42 & 0.021 & -28 & 45 & 41 & 1522 \\
\hline Inferior frontal gyrus & $\mathrm{R}$ & 4.91 & 0.030 & 15 & 21 & 5 & 1733 \\
\hline Middle temporal gyrus & B & 5.92 & 0.006 & 0 & -36 & 14 & 1123 \\
\hline Posterior parietal cortex & B & 5.03 & 0.027 & 0 & -43 & 2 & 1893 \\
\hline Angular gyrus & L & 4.55 & 0.045 & -35 & -37 & 11 & 961 \\
\hline Cerebellum & $\mathrm{R}$ & 5.81 & 0.032 & 8 & -38 & -20 & 3206 \\
\hline \multicolumn{8}{|l|}{ TN woNVC vs. HC } \\
\hline Inferior temporal gyrus & B & 5.99 & 0.021 & 0 & 17 & -23 & 2452 \\
\hline Posterior cingulate cortex & $\mathrm{B}$ & 5.48 & 0.015 & 0 & -14 & 10 & 1842 \\
\hline \multicolumn{8}{|l|}{ TN wNVC vs. TN woNVC } \\
\hline Medial prefrontal cortex & $\mathrm{R}$ & 6.83 & 0.019 & 2 & 9 & 44 & 1723 \\
\hline Cerebellum & L & 5.88 & 0.034 & -4 & -21 & -25 & 2109 \\
\hline
\end{tabular}

That all MNI coordinates of maximum $t$ values are selected in the significant region

That there was no significant difference in gray matter volume between the $S Z$ and $B D$ groups

Abbreviations: SZ Schizophrenia, $B D$ Bipolar disorder, $H C$ Healthy control, MNI Montreal Neurological Institute, $L$ Left, $R$ Right

${ }^{\text {a }}$ Family-wise error corrected $P<0.017$ 

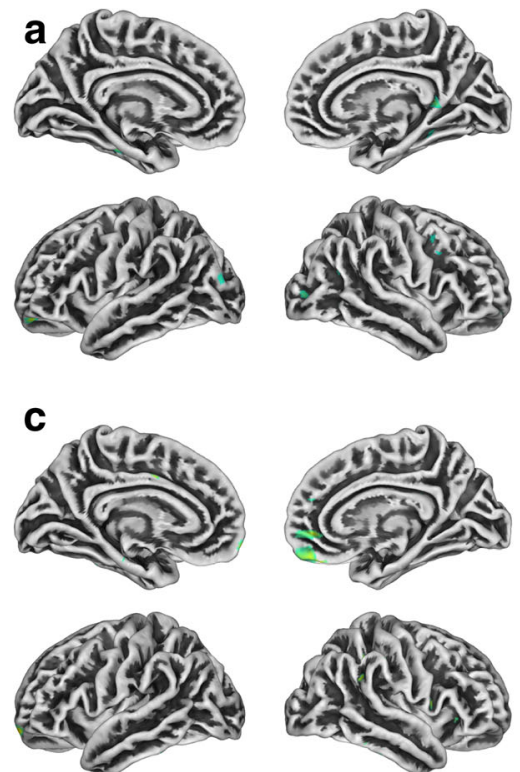

b
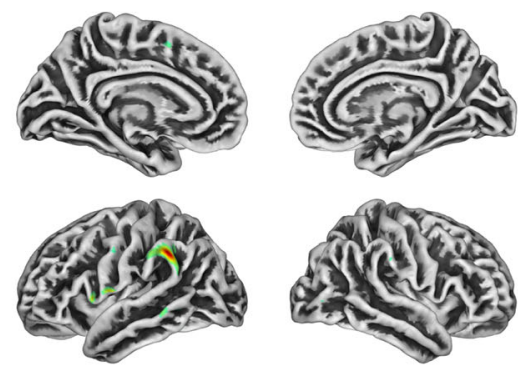

d

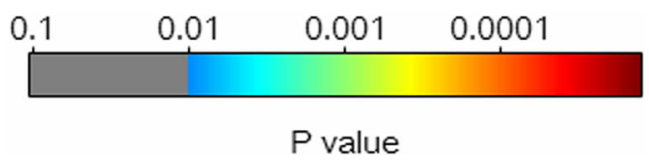

Fig. 2 Results of the surface-based morphometry (cortical thickness) analysis comparing 23 patients with TN wNVC and 22 patients with TN WNVC and 45 healthy controls. Results of the statistical analysis ( $p$ map) are overlaid on a tridimensional rendering of the brain

alterations in diffusion were identified in both the TN wNVC and TN woNVC groups (Tables 4 and 5). Compared to HC, patients with TN wNVC showed FA decreased in bilateral superior corona radiata, right anterior limb of internal capsule, splenium of corpus callosum, body of corpus callosum, right cingulum, and left genu of internal capsule, RD increased in the splenium of the corpus callosum, right anterior limb of internal capsule, left posterior thalamic radiation, and bilateral posterior corona radiata. (Fig. 3a, b) However, patients with TN woNVC showed FA decreased in the bilateral anterior corona radiata, and bilateral fasciculus uncinatus, and RD increased in the splenium of corpus callosum, right anterior thalamic radiation, left anterior limb of internal, left external capsule and bilateral superior corona radiata. Further, direct comparisons of DTI parameters between TN wNVC and TN woNVC revealed no significant differences (Fig. 3c, d).

\section{Topology and hubs organization changes (DTI)}

The measures of global topology in patients with TN wNVC or TN woNVC showed different characteristics, compared with that in HC. As compared with the HC group, patients with TN wNVC had significant lower global efficiency, local efficiency and higher characteristic path length, while patients with TN woNVC had significant higher global efficiency, local efficiency, and lower small-worldness (Table 6). There were significant

Table 3 Pairwise comparisons of surface-based morphometry (SBM) analyses between TN wNVC, TN woNVC, and HC groups

\begin{tabular}{|c|c|c|c|c|c|c|c|}
\hline \multirow[t]{2}{*}{ Anatomical region } & \multirow[t]{2}{*}{ Side } & \multirow[t]{2}{*}{$\mathbf{T}$} & \multirow[t]{2}{*}{$P$} & \multicolumn{3}{|c|}{ Peak coordinates (MNI) } & \multirow{2}{*}{$\begin{array}{l}\text { Cluster } \\
\text { size } \\
\left(\mathrm{mm}^{3}\right)\end{array}$} \\
\hline & & & & $\mathbf{x}$ & y & z & \\
\hline \multicolumn{8}{|l|}{ TN wNVC vs. HC } \\
\hline Inferior frontal gyrus & $\mathrm{L}$ & 4.33 & 0.011 & -12 & 25 & 8 & 1620 \\
\hline Medial frontal gyrus & $\mathrm{R}$ & 4.36 & 0.032 & 21 & 17 & 24 & 1442 \\
\hline Superior occipital gyrus & B & 3.94 & 0.028 & 0 & -53 & 10 & 2371 \\
\hline \multicolumn{8}{|l|}{ TN woNVC vs. HC } \\
\hline Angular gyrus & L & 4.82 & 0.026 & -36 & -48 & 43 & 1363 \\
\hline Medial frontal gyrus & L & 4.72 & 0.003 & -12 & 45 & 12 & 1887 \\
\hline Inferior temporal gyrus & L & 4.40 & 0.006 & -27 & -34 & -14 & 1093 \\
\hline \multicolumn{8}{|l|}{ TN wNVC vs. TN woNVC } \\
\hline Medial prefrontal cortex & B & 4.95 & 0.010 & 0 & 36 & -2 & 1989 \\
\hline
\end{tabular}


Table 4 Comparisons of diffusion indexes between TN wNVC and HC groups

\begin{tabular}{|c|c|c|c|c|c|c|c|}
\hline \multirow[t]{2}{*}{ Anatomical region } & \multirow[t]{2}{*}{ Side } & \multirow{2}{*}{$\begin{array}{l}\mathrm{T} \\
\max \end{array}$} & \multirow[t]{2}{*}{$P$} & \multicolumn{3}{|c|}{ Peak coordinates (MNI) } & \multirow{2}{*}{$\begin{array}{l}\text { Cluster } \\
\text { size } \\
(\mathrm{mm})\end{array}$} \\
\hline & & & & $x$ & $y$ & $\mathbf{z}$ & \\
\hline \multicolumn{8}{|l|}{ FA TN wNVC vs. HC } \\
\hline Superior corona radiata & L & 4.124 & 0.0022 & -31 & -30 & 27 & 218 \\
\hline Superior corona radiata & R & 4.392 & 0.0028 & 28 & -15 & 33 & 304 \\
\hline Anterior limb of internal capsule & $\mathrm{R}$ & 5.920 & 0.0039 & 24 & 26 & 4 & 412 \\
\hline Splenium of corpus callosum & & 4.729 & 0.0030 & -2 & -36 & 31 & 542 \\
\hline Body of corpus callosum & & 5.325 & 0.0043 & 5 & 11 & 19 & 1332 \\
\hline Cingulum & R & 4.782 & 0.0036 & 8 & 14 & 32 & 392 \\
\hline Genu of internal capsule & L & 3.882 & 0.0019 & -18 & 21 & 13 & 430 \\
\hline \multicolumn{8}{|l|}{ RD TN wNVC vs. HC } \\
\hline Splenium of corpus callosum & & 4.644 & 0.0037 & 3 & -29 & 37 & 526 \\
\hline Anterior limb of internal capsule & $\mathrm{R}$ & 5.801 & 0.0040 & 22 & 30 & 8 & 669 \\
\hline Posterior thalamic radiation & L & 5.261 & 0.0035 & -27 & -49 & 21 & 573 \\
\hline Posterior corona radiata & L & 4.602 & 0.0030 & -37 & -52 & 31 & 629 \\
\hline Posterior corona radiata & $\mathrm{R}$ & 3.772 & 0.0017 & 28 & -44 & 29 & 478 \\
\hline
\end{tabular}

differences in the reorganization of hubs organization in both two groups of patients compared to healthy subjects. In the comparison between TN wNVC and $\mathrm{HC}$, the strength of left middle frontal gyrus, left inferior occipital gyrus, right posterior parietal cortex and left angular gyrus in the patients with TN wNVC were lower than those in healthy subjects. In addition, the betweenness centrality of right middle temporal gyrus, right insula, left angular gyrus and left thalamus in the patients with TN wNVC were lower than those in HC. Moreover, the eigenvector centrality of left angular gyrus, right superior frontal gyrus, left posterior parietal gyrus and left middle frontal gyrus in the patients with TN wNVC were lower than those in healthy subjects. In the comparison between TN woNVC and $\mathrm{HC}$, the strength of left inferior temporal gyrus, right posterior cingulate cortex, right hippocampus, left superior temporal gyrus and right inferior frontal gyrus in the patients with TN woNVC were lower than those in HC. Besides, the betweenness centrality of left inferior frontal gyrus, left hippocampus, left inferior temporal gyrus and right posterior cingulate cortex in the patients with $\mathrm{TN}$ woNVC were lower than those in HC. Moreover, the eigenvector centrality of left posterior cingulate cortex, right posterior cingulate cortex and left angular gyrus in the patients with TN woNVC were lower than those in $\mathrm{HC}$.

Table 5 Comparisons of diffusion indexes between TN woNVC and HC groups

\begin{tabular}{|c|c|c|c|c|c|c|}
\hline \multirow[t]{2}{*}{ Anatomical region } & \multirow[t]{2}{*}{ Side } & \multirow{2}{*}{$\begin{array}{l}\mathbf{T} \\
\max \end{array}$} & \multicolumn{3}{|c|}{ Peak coordinates (MNI) } & \multirow{2}{*}{$\begin{array}{l}\text { Cluster } \\
\text { size } \\
(\mathrm{mm})\end{array}$} \\
\hline & & & $x$ & $y$ & z & \\
\hline \multicolumn{7}{|l|}{ FA TN woNVC vs. HC } \\
\hline Anterior corona radiata & L & 5.82 & -31 & 45 & 32 & 662 \\
\hline Anterior corona radiata & $\mathrm{R}$ & 5.91 & 27 & 47 & 30 & 732 \\
\hline Fasciculus uncinatus & L & 4.23 & -42 & 9 & -5 & 403 \\
\hline Fasciculus uncinatus & $\mathrm{R}$ & 4.05 & 38 & 11 & -9 & 437 \\
\hline \multicolumn{7}{|l|}{ RD TN woNVC vs. HC } \\
\hline Splenium of corpus callosum & & 4.665 & 3 & -29 & 37 & 530 \\
\hline Anterior thalamic radiation & $\mathrm{R}$ & 5.023 & 5 & -10 & 14 & 572 \\
\hline Anterior limb of internal capsule & L & 4.032 & -27 & 31 & 16 & 539 \\
\hline External capsule & L & 3.782 & -33 & 26 & 23 & 402 \\
\hline Superior corona radiata & L & 5.820 & -22 & 8 & 43 & 659 \\
\hline Superior corona radiata & $\mathrm{R}$ & 5.521 & 16 & 7 & 37 & 587 \\
\hline
\end{tabular}



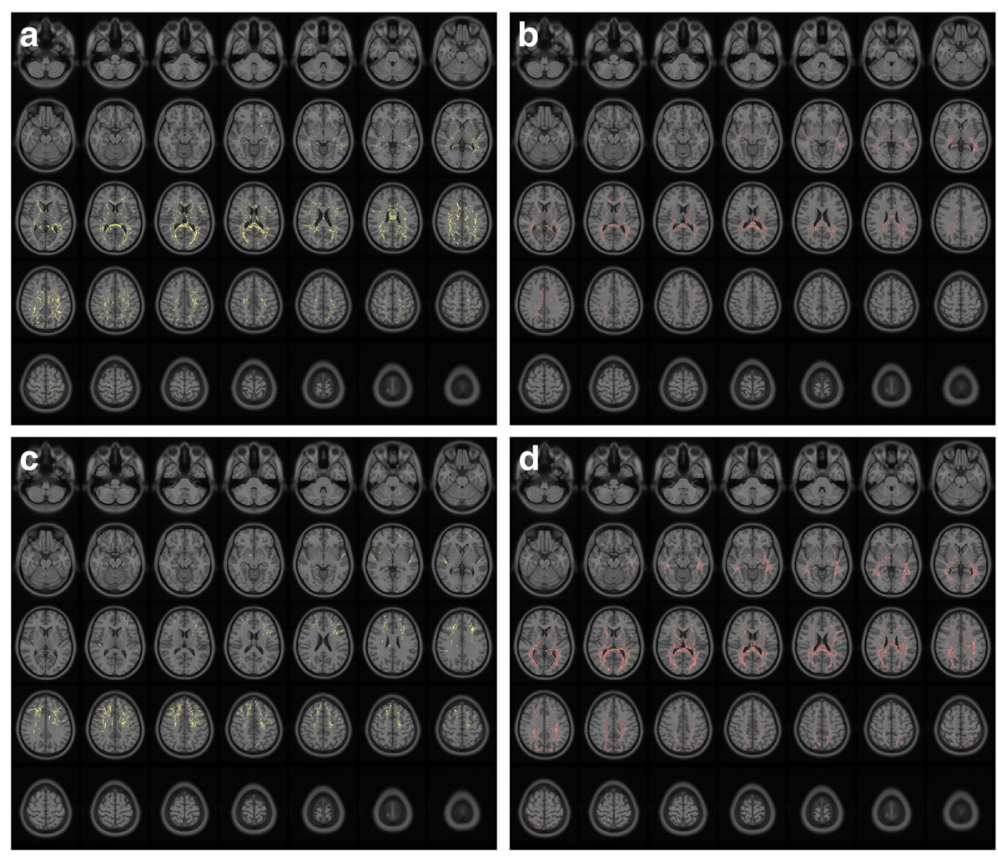

Fig. 3 Results of the diffusivities measures (fractional anisotropy and radial diffusivity) analysis comparing 23 patients with TN wNVC and 22 patients with TN wNVC and 45 healthy controls. Results of the statistical analysis are overlaid on an axial T1 anatomical magnetic resonance imaging template

\section{Intraoperative findings}

The absence or the presence of NVC was verified in a number of 21 patients respectively after complete exploration of the trigeminal root from the porus of the trigeminal Meckel's cave to the trigeminal root entry zone (TREZ) at the pons. The intraoperative findings were recorded and contributed to the diagnosis of $\mathrm{TN}$ wNVC and TN woNVC, combined with preoperative clinical assessment.

\section{Discussion}

The current study is to investigate the associations and differences between variations in brain GM structure (GM volume and cortical thickness), WM integrity (i.e., DTI parameters, global graph theoretical network measures, and hubs organization) and the factor of NVC within a cohort of patients with TN and sex-, agematched HC. Overall, the results obtained are in line with the concept that $\mathrm{TN}$ is associated with GM and WM abnormalities [16], while, there are obviously different manifestations between TN wNVC and TN woNVC. To our knowledge, this is the first study to analyze brain pathophysiology and pathogenetic mechanism in $\mathrm{TN}$ wNVC and TN woNVC, via structural and diffusion MRI.

GM alterations occur in common areas in TN wNVC and TN woNVC but are more widespread in TN woNVC

In this study, compared to $\mathrm{HC}$, in $\mathrm{TN}$ wNVC, the GM volume deficits were widely distributed across the thalamus, superior frontal gyrus, inferior frontal gyrus, middle temporal gyrus, angular gyrus, and posterior central gyrus. These affected regions were mostly consistent with those reported by previously published large-sized VBM analyses and meta-analyses which have reported that patients with CTN show GM volume deficits

Table 6 Comparison of global topographic parameters between TN WNVC and TN woNVC compared with HC

\begin{tabular}{|c|c|c|c|c|c|}
\hline \multirow[t]{2}{*}{ Network measures } & \multicolumn{2}{|l|}{ TN wNVC } & \multicolumn{2}{|l|}{ TN woNVC } & \multirow[t]{2}{*}{$\mathrm{HC}$} \\
\hline & Mean \pm SD & $p$-value & Mean \pm SD & $p$-value & \\
\hline Mean clustering coefficient & $0.2867 \pm 0.0085$ & 0.3486 & $0.2883 \pm 0.0087$ & 0.9256 & $0.2881 \pm 0.0041$ \\
\hline Characteristic path length & $4.2004 \pm 0.2313$ & 0.0022 & $3.6647 \pm 0.5478$ & 0.2681 & $3.8229 \pm 0.5431$ \\
\hline Small-worldness & $0.1891 \pm 0.0076$ & 0.1093 & $0.1825 \pm 0.0135$ & 0.0009 & $0.1932 \pm 0.0108$ \\
\hline Global efficiency & $0.7954 \pm 0.1112$ & 0.0068 & $0.9259 \pm 0.1101$ & 0.0027 & $0.8570 \pm 0.0699$ \\
\hline Local efficiency & $1.1535 \pm 0.1615$ & 0.0235 & $1.7174 \pm 0.5502$ & 0.0013 & $1.3385 \pm 0.3635$ \\
\hline
\end{tabular}


throughout the frontal, temporal and parietal lobes; cingulate and insular cortices; and thalamus [17-23]. In contrast, in patients with TN woNVC as compared with $\mathrm{HC}$, we observed almost all reductions in GM volume were bilateral symmetrical, which were limited to the bilateral inferior temporal gyrus, bilateral PCC. Overall, our VBM analyses could draw the conclusion that TN wNVC and TN woNVC display quite different types of GM volume deficits when compared with $\mathrm{HC}$ group, although share common deficits in the posterior cingulate cortex and inferior temporal gyrus. Meanwhile, in the cortical thickness analysis, left angular gyrus was detected in the comparison between TN woNVC and $\mathrm{HC}$, and right mPFC was significantly decreased in $\mathrm{TN}$ woNVC when compared with TN wNVC. Medial temporal lobe plays a role in sensitization-like processes related to the affective component of pain, which may be linked to impaired memory in fibromyalgia [24]. The association among $\mathrm{TN}$, migraine, cluster headache, and low back pain in epidemiology and pathogenesis has been acknowledged [25-27]. Thalamic-somatosensory dysfunction and dysrhythmia have been proved to be associated with TN, migraine and low back pain, and the abnormal functional connectivity between the thalamus and attentional cerebral networks has been substantially proved at rest during migraine attacks [10, 28-31]. With regard to the $\mathrm{PCC}$, it play an important role in pain modulation, and has been considered as crucial brain regions of default mode network (DMN) [32]. Moreover, the direct comparison between TN wNVC and TN woNVC showed both the GM volume and cortical thickness of right MPC were decreased in TN woNVC. The $\mathrm{mPFC}$ is one of the crucial component in conditioned pain modulation (CPM), especially participating in topdown pain processing $[33,34]$. The mPFC also compose the DMN with PCC, angular gyrus [35], and the TN woNVC can be associated with the changes of these structure according to our findings.

Recent series of studies have identified several cortical and subcortical regions associated with GM volume reduction in patients with TN wNVC $[17,19,20,22,23$, 36, 37]. Putting the findings of these studies together, it can be inferred that TN wNVC is associated with extensive deficits. Our VBM and SBM analyses performed simultaneous comparisons between TN wNVC vs. HC and between TN woNVC vs. $\mathrm{HC}$ and confirmed that GM volume reductions are typically more extensive in TN wNVC than in TN woNVC, and that there is an overlap in the affected regions between the two disorders. In addition, patterns of the overlap between patients with TN wNVC and those with TN woNVC suggest that GM volume and cortical thickness deficits were focused in the medial frontal gyrus, which might be common neurobiological substrates across TN wNVC than in TN woNVC. Finally, in the comparison of GM between TN wNVC and TN woNVC, patients with TN wNVC showed a widespread reduction in GM volume, including insula, somatosensory cortex, and thalamus, which was associated with bottom-up pain processing areas [38]; whereas those with TN woNVC showed limited deficits, such as the PFC, might be associated with the top-down pain processing [39].

\section{Widespread compromise of WM integrity occurs in both TN wNVC and TN woNVC}

A combined interpretation of these DTI parameters may allow us to better characterize the alterations of WM microstructure in TN wNVC and TN woNVC. We observed that compared to $\mathrm{HCs}$, significant decreases in $\mathrm{FA}$ and increases in RD were widespread over areas of the whole-brain WM skeleton in both TN wNVC and TN woNVC. Regarding TN wNVC, decreases in FA were coupled with increases in RD in areas of the corpus callosum, corona radiata and internal capsule, which indicates probable damage to pain integration, cognitiveaffective, and motor functions, also could be relative to compensatory mechanisms for this type of TN $[40,41]$. In addition, increases in RD in the areas of the posterior thalamic radiation, which is suggestive of damage to the microstructural properties of the thalamicsomatosensory. However, TN woNVC showed decreases in FA were located to bilaterally symmetrically in anterior corona radiata and fasciculus uncinatus, while increases in RD in the areas of thalamic radiation, internal capsule, external capsule, corpus callosum and bilateral superior corona radiata. Overall, our findings related to TN wNVC and TN woNVC are in accordance with the results of a recent review of DTI data which showed significant global alterations in corpus callosum, cingulum bundle, coronal radiata, and superior longitudinal fasciculus [16].

Further, in order to investigate the effect of WM tissue alterations on the structural brain network, and reveal insight into potential pathophysiological mechanisms underlying TN at the network level, we applied graph theory to obtain topographic parameters. Brain structural connectivity via graph theory analysis has been demonstrated effective for predicting brain functional complexity of BOLD-fMRI signals [42]. With the results of network analysis on a global scale, different patterns of abnormal connectivity in TN wNVC and TN woNVC were found. In TN wNVC, the brain connections of temporal gyrus, occipital lobe, insula, and thalamus are related to multisensory integration of auditory, visual, olfactory, and somatosensory information [43]. Altered connectivity in these regions may interfere the temporal filtering of nociceptive information in offset analgesia [39], and underlie the psychological disturbances 
including anxiety, fear, and panic in TN patients [41], and sensory, cognitive-affective, and modulatory aspects of pain [30]. However, patients with TN woNVC exhibited higher local network integration in the hippocampus, PFC and angular gyrus as compared to $\mathrm{HC}$, which may undermine the spatial filtering of nociceptive information in CPM, especially the top-down pain processing [39]. We inferred that such changes in network connectivity may be an initial key step in the process that ultimately results in clinical symptoms. Our findings suggest that even without the influence of NVC, TN woNVC may induce alterations in anatomical brain connections, possibly due to alteration of top-down pain processing.

There are several methodological limitations to this study. First, we only investigated 23 patients with TN wNVC and 22 patients with TN wNVC, larger sample sizes may be needed in our future study. Second, further study of the functional connectivity and brain white matter plasticity alterations of TN woNVC still need to be complete in order to verify the result of structural network in this research.

\section{Conclusion}

Overall, our findings suggest that $\mathrm{TN}$ is associated with anatomical changes within various brain structures involved in pain modulation. Whilst our data clearly show that, compared with TN wNVC group, abnormalities in characteristic regions of GM and WM structural network were found in TN woNVC group. Evidence of altered processing CPM raises the possibility of dynamic changes in top-down pain processing areas, which may either trigger or alter the sensitivity of the brain so that an external trigger results in a trigeminal neuralgia attack without the participation of NVC. Future investigations will explore the resting state of brains of TN woNVC, if dynamic changes in brain function overlap certain regions of structural alterations, it is possible to identify promising novel target for we may be in a position to modify these changes and potentially prevent the triggering of $\mathrm{TN}$ woNVC.

\section{Abbreviations \\ BOLD: Blood oxygen level-dependent; CPM: Conditioned pain modulation; DTI: Diffusion tensor imaging; FA: Fraction of anisotropy; HC: Healthy control; IN: Internal neurolysis; MRI: Magnetic resonance imaging; MVD: Microvascular decompression; NVC: Neurovascular compression; RD: Radial diffusivity; SBM: Surface-based morphometry; SF-MPQ: Short-form McGill Pain Questionnaire; TN: Trigeminal neuralgia; TN wNVC: Trigeminal neuralgia with neurovascular compression; TN woNVC: Trigeminal neuralgia without neurovascular compression; VAS: Visual analog scale; VBM: Voxel-based morphometry}

\section{Authors' contributions}

MW, JQ, XFJ: Conceptualization. MW, JQ: Methodology, Software, Validation, Data Curation, Formal analysis. MW, XFJ: Resources. MW, XFJ, JQ: Investigation. MW: Writing - Original Draft, Writing - Review \& Editing, Visualization. MW, XFJ, XMF: Project administration. CSN: Supervision. The author(s) read and approved the final manuscript.

\section{Competing interests}

The authors declare that they have no competing interests.

\section{Author details}

${ }^{1}$ Department of Neurosurgery, The First Affiliated Hospital of USTC (Anhui Provincial Hospital), Division of Life Sciences and Medicine, University of Science and Technology of China, Hefei, Anhui 230001, P.R. China. ${ }^{2}$ Anhui Provincial Stereotactic Neurosurgical Institute, Hefei, Anhui 230001, P.R. China. ${ }^{3}$ Department of Diagnostic Radiology, The First Affiliated Hospital of USTC (Anhui Provincial Hospital), Division of Life Sciences and Medicine, University of Science and Technology of China, Hefei, Anhui 230001, P.R. China.

Received: 30 September 2020 Accepted: 18 November 2020

Published online: 25 November 2020

\section{References}

1. Jannetta PJ (1967) Arterial compression of the trigeminal nerve at the pons in patients with trigeminal neuralgia. J Neurosurg 26(Suppl):159-162

2. Bendtsen L, Zakrzewska JM, Heinskou TB, Hodaie M, Leal PRL, Nurmikko T, Obermann M, Cruccu G, Maarbjerg S (2020) Advances in diagnosis, classification, pathophysiology, and management of trigeminal neuralgia. Lancet Neurol 19:784-796

3. Magown P, Ko AL, Burchiel KJ (2019) The spectrum of trigeminal neuralgia without neurovascular compression. Neurosurgery 85:E553-E559

4. Ko AL, Ozpinar A, Lee A, Raslan AM, McCartney S, Burchiel KJ (2015) Longterm efficacy and safety of internal neurolysis for trigeminal neuralgia without neurovascular compression. J Neurosurg 122:1048-1057

5. Lee A, McCartney S, Burbidge C, Raslan AM, Burchiel KJ (2014) Trigeminal neuralgia occurs and recurs in the absence of neurovascular compression. J Neurosurg 120:1048-1054

6. Jani RH, Hughes MA, Gold MS, Branstetter BF, Ligus ZE, Sekula RF Jr (2019) Trigeminal nerve compression without trigeminal neuralgia: intraoperative vs imaging evidence. Neurosurgery 84:60-65

7. Caston RM, Smith EH, Davis TS, Rolston JD (2020) The cerebral localization of pain: anatomical and functional considerations for targeted electrical therapies. J Clin Med 9:1945

8. Bick SKB, Eskandar EN (2017) Surgical treatment of trigeminal neuralgia. Neurosurg Clin N Am 28:429-438

9. Li M, Yan J, Li S, Wang T, Zhan W, Wen H, Ma X, Zhang Y, Tian J, Jiang G (2017) Reduced volume of gray matter in patients with trigeminal neuralgia. Brain Imaging Behav 11:486-492

10. Rutland JW, Huang KH, Gill CM, Villavisanis DF, Alper J, Verma G, Bederson JB, Delman BN, Shrivastava RK, Balchandani P (2019) First application of 7-t ultra-high field diffusion tensor imaging to detect altered microstructure of thalamic-somatosensory anatomy in trigeminal neuralgia. J Neurosurg:1-9. https://doi.org/10.3171/2019.6.JNS19541

11. Wu M, Qiu J, Jiang X, Li M, Wang SD, Dong Q, Fu X, Niu C (2020) Diffusion tensor imaging reveals microstructural alteration of the trigeminal nerve root in classical trigeminal neuralgia without neurovascular compression and correlation with outcome after internal neurolysis. Magn Reson Imaging 71:37-44

12. Di Stefano G, Maarbjerg S, Truini A (2019) Trigeminal neuralgia secondary to multiple sclerosis: from the clinical picture to the treatment options. J Headache Pain 20:20

13. Arab A, Wojna-Pelczar A, Khairnar A, Szabó N, Ruda-Kucerova J (2018) Principles of diffusion kurtosis imaging and its role in early diagnosis of neurodegenerative disorders. Brain Res Bull 139:91-98

14. Wu M, Fu X, Ji Y, Ding W, Deng D, Wang Y, Jiang X, Niu C (2018) Microvascular decompression for classical trigeminal neuralgia caused by venous compression: novel anatomic classifications and surgical strategy. World Neurosurg 113:e707-e713

15. Wu M, Jiang X, Niu C, Fu X (2018) Outcome of internal neurolysis for trigeminal neuralgia without neurovascular compression and its relationship with intraoperative trigeminocardiac reflex. Stereotact Funct Neurosurg 96: 305-310

16. Moayedi $M$, Hodaie M (2019) Trigeminal nerve and white matter brain abnormalities in chronic orofacial pain disorders. Pain Rep 4:e755

17. Henssen D, Dijk J, Knepfle R, Sieffers M, Winter A, Vissers K (2019) Alterations in grey matter density and functional connectivity in trigeminal neuropathic 
pain and trigeminal neuralgia: a systematic review and meta-analysis. Neurolmage Clin 24:102039

18. Tang Y, Wang M, Zheng T, Yuan F, Yang H, Han F, Chen G (2020) Grey matter volume alterations in trigeminal neuralgia: a systematic review and meta-analysis of voxel-based morphometry studies. Prog NeuroPsychopharmacol Biol Psychiatry 98:109821

19. Wang Y, Yang Q, Cao D, Seminowicz D, Remeniuk B, Gao L, Zhang M (2019) Correlation between nerve atrophy, brain grey matter volume and pain severity in patients with primary trigeminal neuralgia. Cephalalgia 39:515-525

20. Moon HC, Park CA, Jeon YJ, You ST, Baek HM, Lee YJ, Cho CB, Cheong CJ, Park YS (2018) 7 tesla magnetic resonance imaging of caudal anterior cingulate and posterior cingulate cortex atrophy in patients with trigeminal neuralgia. Magn Reson Imaging 51:144-150

21. Wang Y, Zhang Y, Zhang J, Wang J, Xu J, Li J, Cui G, Zhang J (2018) Structural and functional abnormalities of the insular cortex in trigeminal neuralgia: a multimodal magnetic resonance imaging analysis. Pain 159:507-514

22. Tsai YH, Yuan R, Patel D, Chandrasekaran S, Weng HH, Yang JT, Lin CP, Biswal BB (2018) Altered structure and functional connection in patients with classical trigeminal neuralgia. Hum Brain Mapp 39:609-621

23. Obermann M, Rodriguez-Raecke $R$, Naegel $S$, Holle $D$, Mueller D, Yoon MS, Theysohn N, Blex S, Diener HC, Katsarava Z (2013) Gray matter volume reduction reflects chronic pain in trigeminal neuralgia. Neurolmage 74:352-358

24. Nahman-Averbuch H, Martucci KT, Granovsky Y, Weissman-Fogel I, Yarnitsky D, Coghill RC (2014) Distinct brain mechanisms support spatial vs temporal filtering of nociceptive information. Pain 155:2491-2501

25. Lin K-H, Chen Y-T, Fuh J-L, Wang S-J (2016) Increased risk of trigeminal neuralgia in patients with migraine: a nationwide population-based study. Cephalalgia 36:1218-1227

26. Vivekanantham A, Edwin C, Pincus T, Matharu M, Parsons $H$, Underwood M (2019) The association between headache and low back pain: a systematic review. J Headache Pain 20(1):82

27. Vollesen AL, Benemei S, Cortese F, Labastida-Ramírez A, Marchese F, Pellesi L, Romoli M, Ashina M, Lampl C (2018) Migraine and cluster headache - the common link. J Headache Pain 19(1):89

28. Tu Y, Fu Z, Mao C, Falahpour M, Gollub RL, Park J, Wilson G, Napadow V, Gerber J, Chan S-T, Edwards RR, Kaptchuk TJ, Liu T, Calhoun V, Rosen B, Kong J (2020) Distinct thalamocortical network dynamics are associated with the pathophysiology of chronic low back pain. Nat Commun 11:3948

29. Coppola G, Di Renzo A, Tinelli E, Lepre C, Di Lorenzo C, Di Lorenzo G, Scapeccia M, Parisi V, Serrao M, Colonnese C, Schoenen J, Pierelli F (2016) Thalamo-cortical network activity between migraine attacks: insights from mri-based microstructural and functional resting-state network correlation analysis. J Headache Pain 17(1):100

30. Porcaro C, Di Renzo A, Tinelli E, Di Lorenzo G, Parisi V, Caramia F, Fiorelli M, Di Piero V, Pierelli F, Coppola G (2020) Haemodynamic activity characterization of resting state networks by fractal analysis and thalamocortical morphofunctional integrity in chronic migraine. J Headache Pain 21(1):1

31. Coppola G, Renzo AD, Tinelli E, Lorenzo CD, Lorenzo GD, Parisi V, Serrao M, Schoenen J, Pierelli F (2016) Thalamo-cortical network activity during spontaneous migraine attacks. Neurology 87:2154-2160

32. Jones SA, Morales AM, Holley AL, Wilson AC, Nagel BJ (2020) Default mode network connectivity is related to pain frequency and intensity in adolescents. Neurolmage Clin 27:102326

33. Schreiber $\mathrm{KL}$, Loggia ML, Kim J, Cahalan CM, Napadow V, Edwards RR (2017) Painful after-sensations in fibromyalgia are linked to catastrophizing and differences in brain response in the medial temporal lobe. J Pain 18:855-867

34. Chen Q, Heinricher MM (2019) Descending control mechanisms and chronic pain. Curr Rheumatol Rep 21(5):13

35. Cheriyan J, Sheets PL (2018) Altered excitability and local connectivity of mpfcpag neurons in a mouse model of neuropathic pain. J Neurosci 38:4829-4839

36. Hayes DJ, Chen DQ, Zhong J, Lin A, Behan B, Walker M, Hodaie M (2017) Affective circuitry alterations in patients with trigeminal neuralgia. Front Neuroanat 11:73

37. Uddin LQ, Clare Kelly AM, Biswal BB, Xavier Castellanos F, Milham MP (2009) Functional connectivity of default mode network components: correlation, anticorrelation, and causality. Hum Brain Mapp 30:625-637

38. Vaculik MF, Noorani A, Hung PS-P, Hodaie M (2019) Selective hippocampal subfield volume reductions in classic trigeminal neuralgia. Neurolmage Clin 23:101911
39. Andica C, Kamagata K, Hatano T, Saito Y, Ogaki K, Hattori N, Aoki S. MR biomarkers of degenerative brain disorders derived from diffusion imaging. J Magn Reson Imaging. 2020;52(6):1620-36.

40. Ye Q, Chen W, Zhuge Q, Li J, Wang S, Liu H, Wen C, Zhang Z, Xue Y (2019) Characterization of alzheimer's disease using ultra-high b-values apparent diffusion coefficient and diffusion kurtosis imaging. Aging Dis 10:1026

41. Neudorf J, Ekstrand C, Kress S, Borowsky R (2020) Brain structural connectivity predicts brain functional complexity: diffusion tensor imaging derived centrality accounts for variance in fractal properties of functional magnetic resonance imaging signal. Neuroscience 438:1-8

42. Sprenger C, Bingel U, Büchel C (2011) Treating pain with pain: Supraspinal mechanisms of endogenous analgesia elicited by heterotopic noxious conditioning stimulation. Pain 152:428-439

43. Liu J, Zhu J, Yuan F, Zhang X, Zhang Q (2018) Abnormal brain white matter in patients with right trigeminal neuralgia: a diffusion tensor imaging study. J Headache Pain 19:36030

\section{Publisher's Note}

Springer Nature remains neutral with regard to jurisdictional claims in published maps and institutional affiliations.

\section{Ready to submit your research? Choose BMC and benefit from:}

- fast, convenient online submission

- thorough peer review by experienced researchers in your field

- rapid publication on acceptance

- support for research data, including large and complex data types

- gold Open Access which fosters wider collaboration and increased citations

- maximum visibility for your research: over $100 \mathrm{M}$ website views per year

At BMC, research is always in progress.

Learn more biomedcentral.com/submissions 